\title{
Research on the Application of Products based on Unity3D
}

\author{
Lehui Huang1,a,Bin Gui2,b \\ 1Education School, Jiangxi Science\&Technology Normal University, \\ Nanchang, China \\ 2Education School, Jiangxi Science\&Technology Normal University, \\ Nanchang, China \\ aHLH8899@163.com,bguivipbin@qq.com
}

\begin{abstract}
Unity3D is a cross-platform professional game engine which is super hot in recent years. Its graphics, audio and video, lighting, physical effects can simulate the real world environment, making the user feel immersive and consistenting high praise by the game designers. Products produced by Unity3D are more and more widely used in the production and various aspects of life. In this paper, it has been researched on the 3D display, virtual roaming and the application of the simulation system.
\end{abstract}

Keywords: Unity3D; Products; Application

\section{Introduction}

For a long time, games is a very popular industry at home and abroad. With the continuous development of virtual reality technology, equipment and platform, the realistic requirement of the game that people pursuit of is increasing, the game engine also more professional, Unity3D arises at the historic moment. It is an integrated comprehensive professional cross-platform game engine that developed by Danish Unity Technologies[1], which can create 2D and 3D video games, virtual constructions, real-time 3D animations and other types of remarkable interactive effect products. At present, the latest official version is Unity5, still in the escalating, every upgrade greatly boosted its production performance.The engine has simple interface, friendly development environment, high compatibility of a formed complete set of software good interaction running in a variety of platforms, such as Mac, Windows, Android, IOS, Web, Flash, etc. The DirectX and OpenGL graphics rendering pipelines are greatly optimized[2], which made the production process can smoothly on low-end hardware, and be able to create high quality 3D simulation systems, and real visual effects, thus highly favored by the game developers and IT industry personnel, and its products can quickly get the markets.

\section{Application of 3D display}


The most direct and intuitive way to display products is the physical products showed in front of the customer. As technology advances, however, the outbreak of the amount of information, customer's requirement of information collection increased, this old method cannot satisfy the customers. Now the mainstream of display is the use of graphic images with some text, but it is basically still in two-dimensional static form, the acting of the product appearance and characteristics are inadequate. Three-dimensional display, not only can be intuitive comprehensive understanding of the characteristics and appearance of the product, and can let the customer decided the point of view of looking at the product, this kind of interaction is incomparable for two-dimensional way. 3D display is a kind of common application of virtual reality products. Usually in 3D modeling software such as Max, Maya, make models and the corresponding animations, export as FBX file, then import in Unity3D, through scripting languages such as c\#, javascript and UI displaying the animations and the interface. The applications include product sales presentations, students's training and working principle of the learning, etc.

Display products for sale: As now many products sales channels, intense competition, the sales staff should make full use of resources, be integrity and pay attention to strategy, but the most important still is to pay attention to create customer experience, must make people feel real and reliable to arouse consumer's purchase desire. Property roaming[3], as nowadays the latest way for the real estate sales, based on the virtual reality technology, integrated with movie and TV advertising, animation, multimedia and network technology, is a representative of the products show. Through real estate roaming, the way of the most advanced sales, buyers can visually see example image, can also see the community built a few years later based on computer virtual reality (VR) technology in the sales areas, also can watch the surrounding environment design, you can even choose family on the computer. Through virtual product display, it can improve the trade efficiency, reduce costs and facilitate the late maintenance, modify, and save the product.

Applied in training: General education can only provide some basic professional knowledge and the low level of skills[4], if in face of the scale of enterprise development, must carry out multiple skills training to make staff gradually achieve the demand of the continuous development of the enterprise. In order to improve labor productivity and individual career satisfaction, and can directly and effectively serve for the organization, master should continuously take various methods to organize all kinds of personnel training. In companies, enterprises, and schools, training for new employees or students, asking the couple to quickly master the use of a machine or object, sometimes by reason of personnel number or machine, it is inconvenience for each trainee practice in class, in order to guarantee the training effectiveness of the efficient, trainers can train the students through the function of the interaction of virtual product.When teachers teach students to master the use of all kinds of cameras, virtual camera product can show the function of each key, press the button and attach samples taken out after, to make the students can understand clearly, make teachers teaching vivid, and improve students' learning efficiency. 


\section{Application of virtual roaming}

Virtual roaming is an important branch of virtual reality technology[5], its highly immersive, interactive and idea have made it develop rapidly in the game, tourism, construction, medical, aerospace, and many other industry. A typical aspect of virtual roaming is virtual architecture scene roaming, which combined virtual scene building technology with the virtual roaming technology.Virtual roaming is widely applied in making big scenes: campus roaming, street roaming, attractions roaming, game scenes roaming, etc. It is divided into the first person virtual roaming and the third person virtual roaming.

Virtual roaming in the first person: It is basically applied in the scene view and FPS games. Alternative to build the first person in the scene (usually a capsule body), set the radius, properties such as height, weight, move the camera to the top of the alternative to the height of the human eyes in the real world, make the camera to be child-object by binding first-person substitute, assign the code of controlling the direction and rotating to the first person substitute, run the game scene and can control in the scene the camera movement through direction key on the keyboard, so as to realize the virtual roaming in the first person. This virtual roaming can be applied virtual tourism in the real scenic landscape, enables users to never leave home to travel around the world. In regard to the development of attractions of virtual roaming system, credit is China's imperial palace and the west lake scenery virtual tour system. Special representative is the Forbidden City VR project "Son of heaven palace, the Forbidden City", which was completed in 2003. Visitors can use controlling device to roam in the Forbidden City, or switch to flight mode to fly freely in the virtual Forbidden City, close to watch the panorama of the hall of supreme harmony.

Virtual roaming in the third person: It is more image than in the first person, users can clearly observed protagonist with input devices, interactivity enhanced. Compared with the first person virtual roaming, only changed the first person substitutes by visible characters, camera also followed and binding. This is common used in various game scenes, such as RPG (Role - playing Game), ACT (Action Game), AVG (Adventure Game), TPS (Third Personal Shooting Game), FTG (Fighting Game), SPT (Sports Game), RAC (Racing Game), and so on.

\section{Application of making the simulation systems}

With the rapid development of military, science and technology, the simulation has become a necessary method to develop all kinds of complex system, especially in the aerospace field, the development of simulation technology in vehicle and satellite vehicle aspect is indispensable, it can obtain high economic benefit.Simulation refers to repetition the nature of the process in the actual system with the use of models, and study the system of the existence or in the designing through the experiment of system model. When the system is expensive, big experimental risk, or need to be aware of the consequences of the caused by system parameter changing for a long time, the simulation is 
particularly effective research means. The simulation experiment in the simulation system established by Unity3D engine can accelerate the development process and bring great social and economic benefits.In the aviation industry, the simulation technology can make large aircraft designing and the development time shortened by $20 \%$. Pilots can use the ground flight simulator training, not limited to site and climatic conditions, still can save a lot of fuel and money (only about one over ten of the air flight training). In addition, some faults can be set up on the flight simulator training witch unable to set in the air, training pilots to cope with the ability of fault. Another important advantage is its security. Simulation experiment to replace in aerospace industry, can reduce the number of live test by $80 \%$. In the electric power industry, using the simulation system for nuclear power plant debugging, maintenance and troubleshooting, can save a lot of manpower, material and financial resources. Modern simulation technology is not only applied in the field of traditional engineering, but also widely used in society, economy, biology and other areas, such as urban planning, traffic control, resource utilization, pollution control, production management, market forecast, analysis and forecast of the world's economy, population control, etc.. Some difficult to experiment on real systems, such as social and economic system, using the simulation technology has more important significance to study the system.

\section{Application in other aspects}

Unity in other aspects of the application is relatively small, but it's not. Such as education virtual reality system[6] based on the Unity3D, through extensive collection of available graphic, audio and video and animation education teaching material, making model and importing into Unity3D to integrated optimize, through the script realizing interaction, eventually released into the system, teachers and students can take advantage of it to teach and learn. Again, such as the earthquake, tsunami, typhoon, fire, and all kinds of emergency simulation exercise, can prevent disaster and reduce casualties.

\section{Summary}

Unity3D as a new type of professional game engine, witch with powerful function, is not limited to the development of the game. Although at present the application of Unity3D are more focused on the 2D and 3D game producing, but along with the software continuously upgrade, function gradually strengthen, its application in other aspects will gradually in-depth. Unity3D is marked a new epoch in game development, broad prospects, its impact will be profound.

\section{References}

[1]Lebo NiPeng Qi,Lina Yu, Wang Jing. Research and Application of the Virtual

Display Technology based on Unity3D Products. Digital technology and 
application, 2010,09,54 55.

[2]Xingjie Wang, Chunhua Li. 3D Virtual City Research and Application Based on Unity3D Platform. Computer technology and development, 2013, 2013 (23), 241-244.

[3] Huijuan Zhu. Virtual Roaming System based on Unity3D. Computer system application, 2012, 10 (21), $36 \sim 39$.

[4]Lei Cao, Hongtao Dai. Mobile Sales System based on Unity3D Technology. The design and implementation of software, 201401 (35), $40 \sim 42$.

[5]Jinmin Liu, Tieming Ma, Na Wang. Motor Show Virtual Simulation Platform Design based on Unity3D. Journal of heilongjiang August first land reclamation university, 201401 (26), 66-68.

[6]Xiaoqing Chen, Shaowei Wang. The Virtual Reality Technology Application in Education. Software Tribune (education technology), 2011,12,76 78. 